\title{
Heat Transfer Improvement in Heat Exchanger using Porous Medium: a Review
}

\author{
Rajeev Lochan \\ Mechanical Department, Apex \\ Institute of Engineering and \\ Technology, \\ Jaipur, India,
}

\author{
Hari Mohan Sharma \\ Mechanical Department, Apex \\ Institute of Engineering and \\ Technology, \\ Jaipur, India,
}

\author{
Deepak Agarwal \\ Mechanical Department, Apex \\ Institute of Engineering and \\ Technology, \\ Jaipur, India,
}

\begin{abstract}
The present study is to investigate the heat transfer enhancement in a cylindrical heat exchanger using porous media. The heat exchanger is modeled by a cylindrical cavity (Shell) with inlet and outlet thermally insulated ports and five tubes which contain hot water and cold water flows in the shell. The effect of porosity on heat transfer enhancement is studied at the different mass flow rate. The study about the effect of porosity on heat transfer enhancement is done by both experimentally and CFD based and the results are compared with the simple heat exchanger. By decreasing the porosity, the heat transfer rate increases and the mean outlet temperature of the fluid increases for different mass flow rate.
\end{abstract}

\section{Keywords}

Porous medium, Heat exchanger, CFD, porosity, mass flow rate

\section{INTRODUCTION}

Heaters are a part of everyday modern life with a wide range of industrial and engineering applications. Examples include the oil and gas industries, chemical processing, hydrocarbon processing, polymers, pharmaceuticals and food. The efficiency of heaters is an important topic in these devices and provides a new way to design and analyze them. The ideal heater transfers the maximum amount of heat with lowest pressure drop.

In recent years, the high cost of energy and material has led to a dramatic endeavor for designing more efficient and economical heat exchange apparatuses. A great deal of research has been developed to study the different heat transfer enhancement techniques such as using fins and baffles, twisted tapes, coiled tubes as well as adding high conductivity particles to the fluid. Porous medium inserts in internal flows are believed to be one of the promising methods in this field.

\section{LITERATURE REVIEW}

Zhang-Jing Zheng et al. [1] In this research paper the numerical investigation of the heat transfer improvement for convection heat transfer of turbulent fluid flow in a central receiver tube filled with the porous medium under non-uniform circumferential heat flux was carried out. The results are shown that the enhanced receiver tube (ERT) with down-filling porous medium inserts and in-filling porous medium inserts have good thermal enhancement when the ratio of thermal conductivity $(\mathrm{K})$ of the porous medium to working fluid medium $(\lambda \mathrm{s} / \lambda \mathrm{f})$ is less than 1,000 . The Enhanced receiver tubes with out-filling porous inserts and up-filling porous inserts have good thermo-hydraulic performance when $(\lambda \mathrm{s} / \lambda \mathrm{f})$
$>100$. It is observed that the Nusselt number of all kinds of ERTs increases with the increase of Reynolds number, but the Performance Evaluation Criterion decreases with the increase of Re.

Zhang-Jing Zheng et al. [2] In this paper, the numerical investigation on augmentation for convection heat transfer of fluid flow in a solar central receiver tube filled with porous medium and non-uniform circumferential heat flux was carried out. Enhanced receiver tubes (ERTs) of four kinds with different porous insert designs were modeled to optimize the performance of ERT. The results are shown that ERT partially filled with the porous medium has given better heat transfer improvement than that fully filled with porous medium.

N. Targui et al. [3] A numerical study a double pipe heat exchanger with porous structures inserted in the annular gap is made in two configurations: (A) on the inner cylinder and (B) on both the cylinders in a staggered fashion. The flow field is modeled by the Darcy-Brinkman-Forchheimer model for the porous medium. It is found that the highest heat transfer rates are obtained when the porous structures are attached in configuration B particularly at the small spacing and large thicknesses.

Bo-wen Hu et al. [4] In this study, GAHP-TSU presented for the potential solar application like air conditioning and refrigeration systems. In this composite granular solid-liquid PCMs compounded by RT100 and high-density polyethylene with phase change temperature of $100^{\circ} \mathrm{C}$ are piled up as a porous PCMs medium layer. The results are shown that the complete system is almost isothermal at the temperature over $70^{\circ} \mathrm{C}$ and the heat transfer properties are found excellent both for heat absorption and release stages.

Majid Siavashi et al. [5] they explained the simultaneous application of nanoparticles and porous media to enhance heat transfer inside an annulus is investigated numerically. Two-phase mixture model along with Darcye -Brinkmane -Forchheimer relation has been implemented for nanofluid flow simulation in porous media. The results are shown that for design with high permeabilities $(\mathrm{Da}=0.1,0.01), \mathrm{PN}$ has an increasing trend with porous element thickness, while for design with low permeabilities $(\mathrm{Da}=0.0001), \mathrm{PN}$ is found a decreasing trend with porous element thickness and for design with a moderate permeability ( $\mathrm{Da}=0.001)$, an optimum thickness is found corresponds to PN.

Baicun Wang et al. [6] explained the novel design of a GPMfilled pipe structure. It was proposed to improve the heat transfer 
and reduce pressure drop of fluid flowing through the pipes filled with gradient porous materials. It was observed that some typical GPM configurations, were studied with $\mathrm{Rp}=0.6$ and $\mathrm{Rp}=1.0$ and they were showing an enhanced heat transfer and a relatively low friction factor can be reached in comparison with the controls. Velocity-based average pore-size was introduced to explain the reduction in friction factors in GPM configurations.

Fumei Rong et al. [7] observed that the new axisymmetric lattice Boltzmann model for calculation of the fluid flow and heat transfer characteristics in a pipe which filled with porous media. The proposed algorithm is implemented on the Graphics Processing Unit (GPU) using NVIDIA's CUDA for a high calculating speed. It is observed that changing the thickness of the porous media can be significantly improved the heat transfer performance and the effect of porosity is slight both on flow field and temperature field when other parameters are fixed.

Salah Chikh et al. [8] analyzed the performance of an annular heat exchanger with a porous insert for entropy generation rate due to heat transfer and fluid friction for turbulent flow. The porous substrate is attached to the inner pipe. It is investigated that the porous insert with an optimal thickness allows a significant reduction of total entropy generation when it has a high effective thermal conductivity. Hence, this heat transfer enhancement technique has a great merit for thin porous inserts not exceeding about $40 \%$ of the annular gap.

Wonjun Choi and Ryozo Ooka [9] this study experimentally examined the effect of natural convection on Thermal response tests (TRTs) conducted in saturated porous formation. TRTs were conducted with two borehole heat exchangers (BHEs) having the same design but different backfill materials: one is cementgrouted and the other is gravel-backfilled. TRTs were conducted for each BHE at two different heat injection rates (approximately $45 \mathrm{~W} / \mathrm{m}$ and $90 \mathrm{~W} / \mathrm{m}$ ). The results show that when the heat rate was almost doubled, the borehole thermal resistances of the gravel-backfilled and cement-grouted BHEs decreased by $9.8 \%$ and $8.7 \%$, respectively.

Renju Kurian et al. [10] In this study, the efficiency of three liquid-to-gas heat exchangers is experimentally compared. The first one is made of copper tubes embedded in a stack of stainless steel wire meshes; the other one is made of copper tubes having an aluminum foam of porosity 0.94 and the last one, a bare heat changer made of plain copper tubes, all having identical dimensions. Air is used as the external working fluid and distilled water is used as tube side heat transfer fluid. The wire mesh heat exchanger is showed better performance than the metal foam heat exchanger under identical conditions.

\section{HEAT EXCHANGER TYPES}

Heat exchanger is a device which is used for transfer of heat between two pipes which is separated by a wall with one or more than one fluid. In recent years, various types of heat exchangers are used for heat transfer enhancement. Heat exchanger includes the various applications like cooling and heating of fluid flow, evaporation and condensation of fluid, etc.

Heat exchangers are classified by various methods as follows:

1. According to fluid transfer process

2. According to number of fluids

3. According to surface compactness

4. According to construction
5. According to flow arrangements

6. According to heat transfer mechanisms

\section{HEAT TRANSFER TECHNIQUES}

There are various techniques for heat transfer of fluids, explain as follows:

1. Active Method: This method is used for heat transfer enhancement of various heat exchangers. This method is very expensive and complex and it is used in reciprocating plungers and magnetic field etc

2. Passive Method: This method is generally used for heat transfer techniques. In this method, heat transfer can be enhanced by using rough surfaces, extended surfaces, nanofluid concentration mixed with working fluid, fins implementations, twisted tape inserts, vortex flow devices and using porous medium.

3. Compound Method: This method is the combination of above two methods and it has limited applications because it involves complex physics.

\section{HEAT TRANSFER IMPROVEMENT USING POROUS MEDIUM}

Porous medium helps to improve the thermal conductivity of working medium as compared to the thermal conductivity of fluid so local heat transfer coefficient as well as Nusselt number increases. When porous medium is used then flow of fluid becomes laminar due to obstruction in flow. The porous medium is worked on the following principle namely flow redistribution and modification in thermal conductivity. Generally metals chips, concretes and nanofluid with porous medium are used for heat transfer. Various thickness of chips, porous attached to the surface, backfill materials, gravel backfields, aluminum foam etc are used as a porous medium. Figure 1 also explained [5] that porous medium with nanofluid concentration improve the Nusselt number values and due to this heat transfer rate increases.

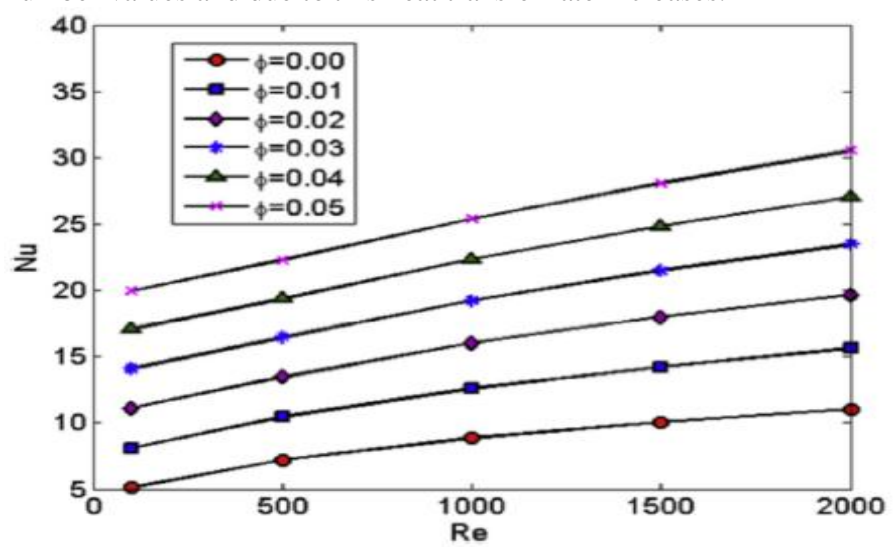

Figure 1: Variation of $\mathrm{Nu}$ with Re for Porous medium using nanofluid

\section{CONCLUSION}

Heat exchangers are widely used as a heat transfer device but use of porous medium enhances the heat transfer rate. Porous medium greatly affect the heat area per unit volume. In this article, a comprehensive review of previous efforts is presented for different convective flow regimes and heat transfer through porous media with and without nanofluid. The effects of several Parameters in porous media geometry and nanofluid properties, thermal boundary conditions, and types of fluids were 
investigated. It is also observed that different types of porous mediums with their different parameters are used for heat transfer enhancement purposes.

\section{REFERENCES}

[1] Zhang-Jing Zheng, Ming-Jia Li, Ya-Ling He, "Optimization of Porous Insert Configuration in a central Receiver Tube for Heat Transfer Enhancement", Energy Procedia, vol. -75, pp. $502-507,2015$

[2] Zhang-Jing Zheng, Ming-Jia Li, Ya-Ling He, "Thermal analysis of solar central receiver tube with porous inserts and non-uniform heat flux", Applied Energy (2015).

[3] N. Targui, H. Kahalerras, "Analysis of fluid flow and heat transfer in a double pipe heat exchanger with porous structures", Energy Conversion and Management, vol.-49, pp. 3217-3229, 2008

[4] Bo-wen Hu, Qian Wang, Zhen-Hua Liu, "Fundamental research on the gravity assisted heat pipe thermal storage unit (GAHP-TSU) with porous phase change materials (PCMs) for medium temperature applications", Energy Conversion and Management, vol.-89, pp. 376-386, 2015

[5] Majid Siavashi, Hamid Reza Talesh Bahrami, Hamid Saffari, "Numerical investigation of flow characteristics, heat transfer and entropy generation of nanofluid flow inside an annular pipe partially or completely filled with porous media using two-phase mixture model", Energy, vol.-93, pp. 24512466,2015
[6] Baicun Wang, Yifeng Hong, Xiangtao Hou, Zhongbin Xu , Pengfei Wang, Xudong Fang, Xiaodong Ruan, "Numerical configuration design and investigation of heat transfer enhancement in pipes filled with gradient porous materials", Energy Conversion and Management, vol.-105, pp. 206-215, 2015

[7] Fumei Rong, Wenhuan Zhang, Baochang Shi, Zhaoli Guo, "Numerical study of heat transfer enhancement in a pipe filled with porous media by axisymmetric TLB model based on GPU", International Journal of Heat and Mass Transfer, vol.-70, pp.1040-1049, 2014

[8] Salah Chikh, Nadia Allouache, "Optimal performance of an annular heat exchanger with a porous insert for a turbulent flow", Applied Thermal Engineering , vol.-104, pp.222-230, 2016

[9] Wonjun Choi, Ryozo Ooka, "Effect of natural convection on thermal response test conducted in saturated porous formation: Comparison of gravel-backfilled and cementgrouted borehole heat exchangers", Renewable Energy, vol.96, pp.891-903, 2016

[10] Renju Kurian, C. Balaji , S.P. Venkateshan, "Experimental investigation of near compact wire mesh heat exchangers", Applied Thermal Engineering, vol.-108, pp.1158-1167, 2016 